\title{
Avaliação do consumo alimentar como preditor de risco para eventos cardiovasculares em jogadores de futebol de várzea
}

\author{
Food consumption evaluation as a predictor of risk for \\ cardiovascular events in várzea football players
}

\author{
Letícia Pereira Pinto ${ }^{1}$ \\ Felipe Hendler De Bem² \\ Caroline Dani ${ }^{3}$ \\ Norberto Garin ${ }^{4}$ \\ Randhall Carteri ${ }^{4}$ \\ Sabrina Alves Fernandes ${ }^{5}$
}

\section{RESUMO}

Introdução: Sabe-se que o futebol de várzea, principalmente na forma recreativa, ou seja, não realizado de forma contínua, torna-se uma prática irregular de exercício físico, que em conjunto com maus hábitos de vida e uma má alimentação torna os seus praticantes expostos a eventos cardiovasculares.Objetivo: Verificar a existência de fatores de risco para eventos cardiovasculares relacionados ao consumo alimentar e composição corporal de jogadores de futebol de várzea. Metodologia: Foram avaliados 17 jogadores do gênero masculino, acima de 18 anos. Para aferição da taxa metabólica basal e avaliação da composição corporal, foi utilizado o aparelho de bioimpedância elétrica (BIA) da marca Bydinamics modelo p450. Foi aferido a circunferência de cintura (CC) e IMC, a partir das medidas de peso e altura. Foi realizado um recordatório alimentar de 24 horas e para cálculo destes, foi utilizado software Avanutriß online. E a frequência de consumo dos grupos alimentares foi analisada através do teste “Como está sua alimentação?”. Além disso, foram calculados o Índice de conicidade e a relação cintura-estatura.Resultados: A alimentação dos jogadores caracterizou-se hiperprotéica (23,75\% do VCT), hipoglicídica (49,45\% do VCT) e normolipídica (27,03\%), porém deficitária a nível de micronutrientes. 0 perfil antropométrico da população foi de sobrepeso em 76,5\% dos participantes, 5,9\% obesos e 16,6\% eutrófico. Também 82,4\% apresentou relação cintura-estatura elevada e 70,6\% dos indivíduos possuíam Índice de conicidade acima do recomendado. Conclusão: Os jogadores analisados apresentaram comprometimento no padrão alimentar e antropométrico, podendo refletir negativamente nas funções vitais dos indivíduos, tornando-os potencialmente suscetíveis a eventos cardiovasculares.

\section{PALAVRAS-CHAVE}

Doenças cardiovasculares; Fatores de risco; Consumo alimentar.

\footnotetext{
${ }^{1}$ Graduada em Nutrição pelo Centro Universitário Metodista - IPA.

${ }^{2}$ Graduado em Educação Física pelo Centro Universitário Metodista - IPA.

${ }^{3}$ Pesquisadora no setor da uva e do vinho auxiliando em projetos diversos e na elaboração de materiais sobre os benefícios dos derivados da uva à saúde humana.

${ }^{4}$ Docente do Centro Universitário Metodista - IPA.

${ }^{5}$ Pesquisadora em Doenças Hepáticas. Experiência em Nutrição hospitalar.
} 


\section{ABSTRACT}

Background: It is known that soccer football mainly in recreational form, that is, it is not carried out continuously, it becomes an irregular practice of physical exercise, which together with bad life habits and a bad diet is caused by its practitioners exposed to cardiovascular events.Objective: To verify the presence of risk factors for cardiovascular events related to food consumption and body composition of soccer players.Methodology: 17 male players, over 18 years old, were applied. For the measurement of basal metabolic rate and body composition assessment, the Bydinamics model p450 electric bioimpedance device (BIA) was used. It was affected by waist circumference (WC) and BMI, based on weight and height measurements. A 24-hour food record was performed and to calculate these, the Avanutri® software was used online. And the frequency of consumption of the food groups was analyzed using the test "How is your food?". In addition, the taper index and a waist-to-height ratio were calculated.Results: The players' diet was characterized as hyperoproteic (23.75\% of VCT), hypoglycidic (49.45\% of VCT) and normolipidic (27.03\%), but deficient in the level of micronutrients. The anthropometric profile of the population was measured in $76.5 \%$ of participants, $5.9 \%$ obese and $16.6 \%$ eutrophic, $82.4 \%$ in relation to high waist height and $70.6 \%$ with taper index above the recommended.Conclusion: the players analyzed, compromised in the dietary and anthropometric pattern, can reflect negatively on the individual's vital functions and making them potentially susceptible to cardiovascular events.

\section{KEYWORDS}

Cardiovascular diseases; Risk factors; Food consumption. 


\section{INTRODUÇÃo}

Segundo a Pesquisa Nacional por Amostra de Domicílios realizada pelo Instituto Brasileiro de Geografia e Estatística (2015), 38,8 milhões de brasileiros praticavam algum tipo de esporte no Brasil, sendo o futebol, a principal modalidade praticada no país, com 39,3\% dos praticantes. Além disso o futebol também foi a modalidade mais praticada em todas as grandes regiões do Brasil destacando-se as Regiões Norte (55,9\%), Nordeste $(48,8 \%)$ e Sul $(35,1 \%)$. Entretanto, $74 \%$ dos participantes desta modalidade não atingem a recomendação de 150 minutos semanais, praticando duas vezes na semana ou menos, sendo que cada partida tem duração média de uma hora. Ainda conforme dados da PNAD, 94\% dos praticantes de futebol são homens, além disso esta modalidade concentra $41,4 \%$ dos praticantes de esporte com idades entre 25 e 39 anos. (IBGE, 2015)

Conforme o Vigilância de Fatores de Risco e Proteção para doenças crônicas por Inquérito Telefônico2019 (MINISTÉRIO DA SAÚDE, 2019), a frequência de consumo regular e adequado de frutas e hortaliçasfoi menor em homens $(27,9 \%)$ do que em mulheres $(39,8 \%)$ e a de refrigerantes e bebida alcoólica, maior em homens, do que em mulheres. Além disso, aproximadamente $55,6 \%$ dos homens com idades entre 25 e 34 anos e $63 \%$ dos homens entre 35 a 44 anos têm excesso de peso e pouco mais de $23 \%$ tem obesidade

Conforme estatísticas do cardiômetro, somente no ano de 2017, cerca de 383.961 pessoas faleceram por doenças cardiovasculares, aproximadamente $20 \mathrm{mil}$ óbitos a mais do que no ano de 2016, no qual tiveram aproximadamente 349.938 óbitos (SBC, 2015).

Inúmeros fatores de risco contribuem para doenças cardiovasculares, entre eles o excesso de peso, hipertensão, diabetes, alcoolismo, tabagismo, sedentarismo/ prática irregular de exercício, entre outros. 0 futebol de várzea, quando praticado menos de 50 minutos/dia em frequência inferior a dois dias por semana, é considerado uma prática de exercício irregular, pois não propicia benefício cardiorrespiratório (SCERJ, 2013; TOMÁS; BRITO, 2017).

Comumente, a característica corporal desses jogadores é classificada como sobrepeso e obesidade, reflexo de maus hábitos alimentares, tanto quantitativamente quanto qualitativamente (MENDONÇA; FARIAS;
GUERREIRO, 2016; PONTES; SOUSA; LIMA, 2006; SIPPEL, 2014).

Sendo assim, se faz necessária a avaliação do consumo alimentar de jogadores de futebol de várzea como preditor de risco cardiovascular, visto que esta prática, quando irregular e em conjunto com demais fatores de risco, potencializam as chances de eventos cardiovasculares.

\section{MÉTODOS}

\section{Caracterização da amostra}

Trata-se de um estudo transversal, no qual a população estudada foi composta por jogadores de futebol de várzea de uma equipe de Três Cachoeiras, no estado do Rio Grande do Sul. 0 estudo foi aprovado pelo Comitê de Ética e Pesquisa do Instituto Porto Alegre da Igreja Metodista (IPA), sob parecer do CEP número 1.846.123. Os indivíduos foram convidados a participar do estudo e assinaram o Termo de Consentimento Livre e Esclarecido (TCLE) conforme resolução 466/2012. Foram incluídos no estudo indivíduos do sexo masculino acima de 18 anos, que praticassem futebol aos finais de semana há mais de 6 meses vinculados a equipe máster da cidade de Três Cachoeiras. Indivíduos que apresentassem alguma cardiopatia ou histórico de evento cardiovascular foram excluídos do estudo. A amostra total foi composta de 17 jogadores com idades entre 26 e 59 anos.

\section{Avaliação antropométrica}

Para avaliação nutricional, foram aferidas medidas de peso, estatura e circunferência abdominal. 0 peso foi aferido através da balança eletrônica 0 mron® com capacidade total de $180 \mathrm{~kg}$ e a altura foi mensurada através do estadiômetro da marca Cescorf® com capacidade total de três metros. Posteriormente, com a coleta destas medidas, foi calculado o Índice de Massa Corporal através da Equação 1, proposta por Quételet (1870), e classificado conforme a classificação estabelecida pela Organização Mundial da Saúde (OMS): Magreza grau III $(<16 \mathrm{~kg} / \mathrm{m} 2)$, Magreza grau II (16-16,9 kg/m²), Magreza grau I $\left(17-18,4 \mathrm{~kg} / \mathrm{m}^{2}\right)$, eutrofia $\left(18,5\right.$ a $\left.24,9 \mathrm{~kg} / \mathrm{m}^{2}\right)$, sobrepeso (25,0 a 29,9 kg/m²), obesidade grau I (30,0 a $\left.34,9 \mathrm{~kg} / \mathrm{m}^{2}\right)$, obesidade grau II $\left(35,0\right.$ a $\left.39 \mathrm{~kg} / \mathrm{m}^{2}\right)$, obesidade grau III ( $>40 \mathrm{~kg} / \mathrm{m}^{2}$ ) (WORLD HEALTH ORGANIZATION, 1998). 


$$
I M C=\frac{\text { peso }(\mathrm{kg})}{\text { estatura }^{2}(\mathrm{~m})} \quad \text { Equação } 1
$$

A circunferência da cintura foi efetuada com uso da trena antropométrica da marca Cescorf® através do ponto médio entre a última costela e a crista ilíaca (WORLD HEALTH ORGANIZATION, 1998).

Para definição da obesidade abdominal, foi utilizado o ponto de corte da circunferência cintura para o risco cardiovascular aumentado em homens (>94 cm) (WORLD HEALTH ORGANIZATION, 1998).

\section{Avaliação da composição corporal}

A fim de avaliar o percentual de massa gorda (MG\%) e a massa magra (MM\%),foi realizada bioimpedância elétrica através do aparelho tetrapolar da marca Bydinamics modelo $\mathrm{P} 450 \AA$. Para realizar a avaliação por bioimpedância elétrica, seguiu-se as orientações de uso do fabricante do aparelho, foi solicitado aos participantes que retirassem calçados, meias e adornos como relógio, corrente e pulseira. 0 participante ficou em decúbito dorsal, em posição confortável e relaxado, com os ca com o dorso voltado para cima. Foram utilizados quatro eletrodos da marca Skintak®, os quais foram posicionados da seguinte forma: um eletrodo distal na base do dedo médio do pé direito e o eletrodo proximal um pouco acima da linha da articulação do tornozelo, entre os maléolos medial e lateral, um eletrodo distal na base do dedo médio da mão direita e o eletrodo proximal um pouco acima da linha da articulação do punho direito, coincidindo com processo estiloide.

Para realização do exame o tempo gasto 5 minutos, afim de realizar o mesmo, o avaliado deveria previamente realizaro jejum de pelo menos $4 \mathrm{~h}$, não ter ingerido bebida alcoólica $48 \mathrm{~h}$ antes do exame, nos dois dias que antecedemo exame, evitar alimentos ricos em cafeína (café, chás e chocolate), 24h antes do exame não realizar atividade física e nem sauna, e urinar até $30 \mathrm{mi}-$ nutos antes do teste. Todo preparo foi informado aos participantes 7 dias antes da realização do teste. (KYLE, 2004; ELLIS, 1999)

\section{Adiposidade abdominal}

A adiposidade abdominal foi mensurada através da Relação Cintura Estatura (RCE) e o risco cardiovascular

através do Índice de conicidade (IC). Para cálculo da RCE foi utilizada a Equação 2 sendo o ponto de corte 0,50.

$$
R C E=\frac{\text { Peso Corporal }(\mathrm{kg})}{\text { Altura }(\mathrm{cm})} \quad \text { Equação } 2
$$

Para estabelecimento do IC foi utilizada a Equação 3 , proposta por Valdez.0 ponto de corte utilizado foi 1,25 (PITANGA, 2011).

$I C=\frac{\text { Circunferência Cintura }(\mathrm{m})}{0,109 \times \sqrt{\frac{\text { PesoCorporal }(\mathrm{kg})}{\text { Estatura }(\mathrm{m})}}}$ Equação 3

\section{Avaliação do consumo alimentar}

No momento da avaliação antropométrica realizada, a avaliação do consumo alimentar dos participantes através de um recordatório alimentar de 24 horas e. para cálculo deste registro alimentar foi utilizado o software Avanutri® online. Ao realizar o recordatório alimentar foi utilizado um livro ilustrativo de medidas caseiras "Alimentos Caseiros e suas Porções". A frequência de consumo dos grupos alimentares foi analisada através do teste “Como está a sua alimentação?" do Guia alimentar de bolso Para elaboração do banco de dados e posterior análise estatística foi utilizado o Microsoft Excel, 2013® (MONEGO, 2013; MINISTÉRIO DA SAÚDE et al., 2014).

\section{Análise estatística}

As variáveis quantitativas foram descritas por média e desvio padrão ou mediana e amplitude interquartílica. As variáveis qualitativas foram descritas por frequências absolutas e relativas. As associações foram realizadas pelo teste qui-quadrado de Pearson em conjunto com a análise dos resíduos ajustados.

O nível de significância adotado foi de $5 \%(\mathrm{p}<0,05)$ e as análises foram realizadas no programa SPSS versão 21.0.

\section{RESULTADOS}

O presente estudo avaliou 17 indivíduos do sexo masculino praticantes de futebol amador com média de idade de 39,70 anos $\pm 10,12$. 0 peso médio dos participantes foi de $79,48 \mathrm{~kg}( \pm 7,62)$ e altura média de $171,71 \mathrm{~cm}( \pm 5,08)$. De acordo com os resultados, $76,6 \%$ da população estudada apresentavam IMC de sobrepeso, conforme observado na Tabela 1 . 
Tabela 1. Caracterização da amostra

\begin{tabular}{|c|c|}
\hline Variáveis & $\mathbf{n}=\mathbf{1 7}$ \\
\hline Idade (anos) - média \pm DP & $39,7 \pm 10,1$ \\
\hline Peso (kg) - média \pm DP & $79,5 \pm 7,6$ \\
\hline Altura $(\mathrm{m})-$ média $\pm \mathrm{DP}$ & $1,72 \pm 0,05$ \\
\hline $\mathrm{IMC}\left(\mathrm{kg} / \mathrm{m}^{2}\right)-$ média $\pm \mathrm{DP}$ & $27,1 \pm 3,2$ \\
\hline \multicolumn{2}{|l|}{ Classificação do IMC - n (\%) } \\
\hline Eutrofia & $3(17,6)$ \\
\hline Sobrepeso & $13(76,5)$ \\
\hline Obesidade & $1(5,9)$ \\
\hline Tabagista - n (\%) & $0(0,0)$ \\
\hline Considera alimentação saudável - n (\%) & $8(47,1)$ \\
\hline História familiar de doença & $11(64,7)$ \\
\hline cardiovascular $-\mathrm{n}(\%)$ & \\
\hline Toma medicação - n (\%) & $5(29,4)$ \\
\hline Tem problema de saúde - n (\%) & $4(23,5)$ \\
\hline \multicolumn{2}{|l|}{ Bebida alcoólica - n (\%) } \\
\hline Consome & $14(82,4)$ \\
\hline Não consome & $3(17,6)$ \\
\hline \multicolumn{2}{|l|}{ Atividade física - n (\%) } \\
\hline Não & $7(41,2)$ \\
\hline Sim & $8(47,1)$ \\
\hline 2 a 4 x/semana & $2(11,8)$ \\
\hline
\end{tabular}

Resultados expressos por média \pm desvio padrão, mediana \pm desvio padrão ou n (\%);

* Teste t não pareado;

IMC: Índice de Massa Corporal.

Fonte: Autores (2018).

A média de circunferência de cintura foi de $92,6 \mathrm{~cm}$ $( \pm 11,1)$, entretanto $58,8 \%$ dos participantes apresentavam circunferência de cintura acima do recomendado para indivíduos saudáveis $(94 \mathrm{~cm})$. A média de relação cintura-estatura foi de $0,54( \pm 0,7)$, sendo que $82,4 \%$ apresentava este coeficiente acima do recomendado $(0,50)$, o que demonstra um maior acúmulo de gordura na região abdominal. Em relação ao Índice de conicidade (IC), a média foi de 1,26 $\pm 0,12$, sendo que $70,6 \%$ dos indivíduos apresentavam o IC acima do recomendado $(\leq 1,25)$. Quanto ao percentual de gordura, 52,9\% dos participantes apresentavam o percentual de gordura acima dos valores estabelecidos para indivíduos saudáveis (Tabela 2) (WORLD HEALTH ORGANIZATION, 1998).
Tabela 2. Medidas de composição corporal

\begin{tabular}{lc}
\hline \multicolumn{1}{c}{ Variáveis } & $\mathrm{n}=1$ \\
\hline CC $(\mathrm{cm})$ - média \pm DP & $92,6 \pm 11,1$ \\
CC aumentada $(\geq 94 \mathrm{~cm})-\mathrm{n}(\%)$ & $10(58,8)$ \\
RCE - média \pm DP & $0,54 \pm 0,07$ \\
RCE aumentada $(\geq 0,5)-\mathrm{n}(\%)$ & $14(82,4)$ \\
IC - média \pm DP & $1,26 \pm 0,12$ \\
IC aumentado ( $\geq 1,25)-\mathrm{n}(\%)$ & $12(70,6)$ \\
Massa magra (\%) - média \pm DP & $79,4 \pm 5,7$ \\
Massa gorda (\%) - média \pm DP & $20,6 \pm 5,7$ \\
Massa gorda elevada $\geq 20 \%$ - n( $\%)$ & $9(52,9)$ \\
Taxa Metabólica Basal (Kcal) & $1959 \pm 98,0$ \\
- média \pm DP & \\
Água extracelular - média \pm DP & $38,1 \pm 2,6$ \\
Água intracelular - média \pm DP & $61,9 \pm 2,6$
\end{tabular}

Resultados expressos por média \pm desvio padrão, mediana \pm desvio padrão ou n (\%).

Fonte: Autores (2018).

De acordo com as recomendações das OMS, o consumo alimentar da população estudada foi caracterizado por uma alimentação hiperprotéica, hipoglicídica, normolipídica e hipocalórica quando comparada a taxa metabólica basal.

No recordatório alimentar de 24 horas, pode-se observar que a mediana de consumo de vitamina $\mathrm{D}$ bem como a de vitamina B12 e vitamina $\mathrm{C}$ foi abaixo do recomendado, de acordo com a Dietary Reference Intakes. Aproximadamente $50 \%$ dos indivíduos apresentava uma elevada ingestão de gordura saturada $(8,41 \%$ do VET) e adequada de gorduras monoinsaturada (Tabela 3) (FOOD AND NUTRITION BOARD, 2011).

Apenas 29,4\% dos participantes consumiam as recomendações diárias de frutas e 11,8\% de verduras e legumes. Por outro lado, o consumo de feijão por pelo menos duas vezes todos dias foi realizado por $88,4 \%$ dos indivíduos. 0 consumo de peixes duas ou mais vezes por semana foi realizado apenas por $11,8 \%$ da população enquanto $70,6 \%$ consumia apenas de uma a quatro vezes ao mês. 0 consumo da gordura aparente das carnes era realizado por $64,7 \%$ da população. 
Tabela 3. Dados sobre o recordatório alimentar

\begin{tabular}{|c|c|c|}
\hline Variáveis & $\mathrm{n}=17$ & Recomendações DRIS \\
\hline Carboidratos (\%) - média \pm DP & 49,47 & $55-60 \%$ \\
\hline Proteínas (\%) - média \pm DP & 23,72 & $10-15 \%$ \\
\hline Lipídios (\%) - média \pm DP & 27,03 & $15-30 \%$ \\
\hline Vit. D $(\mu \mathrm{g})$ - mediana (P25-P75) & $1,65(0,22-4)$ & 5 \\
\hline Vit. B12 ( $\mu \mathrm{g})$ - mediana (P25-P75) & $1,44(0,32-2,15)$ & 2,4 \\
\hline Vit. C (mg)- mediana (P25-P75) & $7,70(3,61-17,60)$ & 90 \\
\hline Vit. E (mg)- mediana (P25-P75) & $7,68(3,89-13,02)$ & 15 \\
\hline Cálcio (mg)- mediana (P25-P75) & $384,78(233,19-716,81)$ & 1000 \\
\hline Fósforo $(\mathrm{mg})-$ média $\pm \mathrm{DP}$ & 915,68 & 700 \\
\hline Magnésio (mg) - mediana (P25-P75) & 162,27 & 420 \\
\hline Ferro (mg)- média \pm DP & 15,89 & 8 \\
\hline Zinco (mg)- média \pm DP & 9,11 & 11 \\
\hline Selênio ( $\mu \mathrm{g})$ - mediana (P25-P75) & 41,05 & 55 \\
\hline Potássio (mg) - mediana (P25-P75) & $1812,26(1157,69-27,90,40)$ & 4700 \\
\hline Sódio (mg)- média \pm DP & $2288,36(1900,95-2956,84)$ & $1500-2000$ \\
\hline Colesterol (mg) - mediana (P25-P75) & $245,94(146,8-420,69)$ & 200 \\
\hline G. Saturada (mg) - média \pm DP & $16,57(8,41 \%)$ & $<7 \%$ \\
\hline $\begin{array}{l}\text { G. Poli-insaturada (g)- mediana } \\
\text { (P25-P75) }\end{array}$ & $4,09(2,47-7,90)(2,07 \%)$ & $<10 \%$ \\
\hline G. Monossaturada (g)- média \pm DP & $10,92(5,54 \%)$ & $<20 \%$ \\
\hline Fibras (g) - mediana (P25-P75) & $13,45(10,10-24,11)$ & 38 \\
\hline Kcal - média \pm DP & 1772,76 & $N A^{* *}$ \\
\hline
\end{tabular}

Resultados expressos por média \pm desvio padrão, mediana \pm desvio padrão ou n (\%). NA: Não se aplica. Recomendações retiradas da Dietary Reference Intakes(FOOD AND NUTRITION BOARD, 2011). Fonte: Autores (2018).

Ao avaliar o consumo de alimentos pertencentes ao grupo de cereais, pode-se observar que os participantes consumiam aproximadamente quatro porções/dia de cereais sendo o recomendado seis porções/dia. Apenas $11,8 \%$ dos participantes apresentavam a ingestão de leite e derivados dentro do recomendado de três porções/dia sendo que a grande parte da população consumia 1 porção ou menos de leite e/ou derivados.

$\mathrm{Na}$ análise de grau de processamento, o consumo de frituras, salgadinhos e/ou embutidos era realizado de duas a três vezes por semana por $47,1 \%$, enquanto nenhum participante consumia abaixo de duas vezes na semana. A ingestão de doces e guloseimas era consumido diariamente por apenas 5,9\% e de duas a três vezes por semana por $11,8 \%$ dos participantes.

0 consumo hídrico apresentado por $100 \%$ dos participantes foi abaixo do recomendado (oito copos ou mais/ dias), sendo que $58,8 \%$ consumia menos da me- tade da recomendação diária (quatro copos/ dia), porém o consumo de bebida alcoólica foi predominantemente de uma a seis vezes por semana por $64,8 \%$.

A prática de exercício físico (pelo menos 150 minutos semanais) era realizada por $11,8 \%$ dos participantes, enquanto $41,2 \%$ não praticava nenhum tipo de atividade física e $47 \%$ praticava, porém de forma irregular, ou seja, abaixo do recomendado.

\section{DISCUSSÃo}

Sabidamente, eventos cardiovasculares ocorrem por diversos fatores, dentre eles, o excesso de peso, a prática irregular e a idade, sendo que este último ocorre com o avançar da idade, por volta dos 50 anos quando o organismo passa por alterações morfológicas e funcionais (WORLD HEALTH ORGANIZATION, 2015; DOS SANTOS et al. 2011). 
Tabela 4. Dados sobre o questionário de “Como está a sua alimentação?”

\begin{tabular}{|c|c|c|c|}
\hline Variáveis & $\begin{array}{l}\mathrm{n}=17 \\
\mathrm{n}(\%)\end{array}$ & Variáveis & $\begin{array}{l}\mathrm{n}=17 \\
\mathrm{n}(\%)\end{array}$ \\
\hline Frutas & & Frituras e/ou salgadinhos - n(\%) & \\
\hline Não consume & 11,8 & Raramente/nunca & 23,5 \\
\hline 3 ou mais unidades & 29,4 & Todos os dias & 23,5 \\
\hline 2 unidades & 23,5 & 2 a 3 vezes por semana & 47,1 \\
\hline 1 unidade & 35,3 & 4 a 5 vezes por semana & 5,9 \\
\hline Legumes e Verduras & & Doces e guloseimas & \\
\hline 3 ou menos colheres de sopa & 29,4 & Raramente/nunca & 35,3 \\
\hline 4 a 5 colheres de sopa & 52,9 & $<2$ vezes por semana & 35,3 \\
\hline 6 a 7 colheres de sopa & 5,9 & 2 a 3 vezes por semana & 11,8 \\
\hline 8 ou mais colheres de sopa & 11,8 & 4 a 5 vezes por semana & 5,9 \\
\hline Feijão & & Todos os dias & 11,8 \\
\hline 2 ou mais colheres de sopa por dia & 82,4 & Tipo de gordura utilizado (\%) & \\
\hline 1 colher de sopa ou menos por dia & 17,6 & Banha ou manteiga & 11,8 \\
\hline Carne & & Óleo: soja, girassol, algodão ou ] & 88,2 \\
\hline Não consome & 5,9 & canola & \\
\hline 1 pedaço/dia & 17,6 & Adição de sal em alimentos após servidos & \\
\hline 2 pedaços/dia & 35,3 & no prato $(\%)$ & 118 \\
\hline Mais de 2 pedaços / dia & 41,2 & NIm & 11,8 \\
\hline Arroz (colheres de sopa) - mediana & $5(3,5-7)$ & Copos de água ingeridos por dia (\%) & 00,2 \\
\hline $\begin{array}{l}\text { (P25-P75) } \\
\text { Pães (unidades por dia) - mediana }\end{array}$ & $2(1,5-4)$ & $<4$ copos $/$ dia & 58,8 \\
\hline (P25-P75) & & 4 a 5 copos / dia & 41,2 \\
\hline Bolos (número de fatias) - mediana & $0(0-1)$ & Bebida Alcóolica (\%) & \\
\hline $\begin{array}{l}\text { (P25-P75) } \\
\text { Biscoitos (unidades por dia) - mediana }\end{array}$ & $0(0-2)$ & Diariamente & 11,8 \\
\hline $\begin{array}{l}\text { (P25-P75) } \\
\text { Gordura aparente das carnes - n(\%) }\end{array}$ & & $\begin{array}{l}1 \text { a } 6 \text { vezes na semana } \\
\text { Eventualmente/ raramente }\end{array}$ & $\begin{array}{c}64,7 \\
5,9\end{array}$ \\
\hline Sim & 35,3 & Não consome & 17,6 \\
\hline Não & 64,7 & Atividade física & \\
\hline Peixe - n(\%) & & Não realiza & 41,2 \\
\hline Não consome & 5,9 & Sim (30 minutos todos os dias) & 11,8 \\
\hline Somente algumas vezes ao ano & 11,8 & 30 minutos de 2 a 4 vezes por & 47,1 \\
\hline 2 ou mais vezes por semana & 11,8 & semana & \\
\hline 1 a 4 vezes por mês & 70,6 & Lê informação nutricional & \\
\hline Leite e derivados - n(\%) & & Nunca & 70,6 \\
\hline Não consome & 17,6 & Quase nunca & 17,6 \\
\hline 3 ou mais copos/fatias & 11,8 & & \\
\hline 2 copos/fatia/porções & 5,9 & Algumas vezes & 11,8 \\
\hline$<1$ copo/fatia/porção & 64,7 & \multirow{4}{*}{$\begin{array}{l}\text { Resultados expressos em n (\%). } \\
\text { Fonte: Autores (2018). }\end{array}$} & \\
\hline Tipo de leite - n(\%) & & & \\
\hline Integral & 76,5 & & \\
\hline Semi desnatado/light & 17,6 & & \\
\hline
\end{tabular}


A caracterização da população estudada se assemelha a população no estudo de Mendonça, Farias e Guerreiro (2016) ao avaliarem 15 jogadores de futebol amador com idades entre 25 e 41 anos. Eles perceberam que os mesmos apresentavam média de IMC de $29,46 \mathrm{Kg} / \mathrm{m}^{2}$ $\pm 4,67$, caracterizando sobrepeso em $40 \%$ dos participantes, $13,4 \%$ tinham obesidade grau I e $26,6 \%$ obesidade grau II. Segundo Blüher (2019), os níveis de obesidade triplicaram desde 1975 e mudanças na alimentação global, juntamente com o aumento do sedentarismo, parecem ser os principais fatores da pandemia da obesidade.

Luo et al (2019) ao avaliarem 3.598 indivíduos, com idade entre 35 e 74 anos, com o objetivo de detectar efeitos sinérgicos entre a bebida alcoólica, obesidade abdominal e doenças cardiovasculares, concluíram que o consumo de álcool está significativamente associado $(\mathrm{p}<0,0001)$ com a obesidade abdominal. No presente estudo 82,4\% dos participantes faziam consumo de álcool, sendo que $64,7 \%$ ingeriam de uma a seis vezes por semana e $11,8 \%$ consumia diariamente.

A média de circunferência de cintura foi de 92,6 cm $( \pm 11,1)$, entretanto, ao avaliar individualmente as medidas aferidas, observou-se que 58,8\% dos participantes apresentavam circunferência de cintura acima do recomendado. 0 aumento da circunferência de cintura está diretamente relacionado com o declínio da aptidão cardiorrespiratória, que por sua vez está relacionada com a síndrome metabólica, o aumento da pressão arterial e a hipercolesterolemia (DYRSTAD, 2019).

Segundo Pitanga e Lessa (2007), o Índice de Massa Corporal, Circunferência de cintura (CC), a Relação cintura-quadril e o Índice de conicidade (IC) estão fortemente associados com a Relação cintura-estatura. Entretanto, um outro estudo realizado por Motamed et al (2015) com 3.179 indivíduos entre 40 e 79 anos revelou que o Índice de conicidade possui maior acurácia para eventos cardiovasculares em dez anos quando relacionado a outros índices de obesidade. Na população estudada, 70,6\% dos indivíduos estavam com o IC acima da recomendação para homens $(\leq 1,21)$ e $82,4 \%$ apresentavam CC aumentada $(\leq 0,50)$.

Castanho et al (2013) ao realizarem uma coorte prospectiva com 636 indivíduos concluíram que o consumo de frutas é considerado um fator protetor para alterações na CC, contribuindo para a prevenção da síndrome metabólica. Além disso, uma maior ingestão ve- getais e leguminosas também estão inversamente associadas a doença cardiovascular importante e infarto agudo do miocárdio. Em nosso estudo, somente 29,4\% dos participantes consumiam o número de porções de frutas recomendadas e 11,8\% consumiam as porções de vegetais recomendadas (MILLER et al., 2017).

O consumo apresentado por $41,2 \%$ da população foi acima de duas porções de carne/dia. 0 alto consumo de carne vermelha não tem demonstrado risco para eventos cardiovasculares, entretanto o consumo excessivo de ferro hematínico presente em alimentos de origem animal, principalmente em carnes vermelhas ocasiona o aumento de ferritina, que é considerado fator de risco para doença cardiovascular. Um dos fatores que oferecem fator de risco para eventos cardiovasculares é a gordura aparente das carnes e os conservantes no caso das processadas (MINISTÉRIO DA SAÚDE et al., 2014).

Um estudo realizado por Dehghan et al (2018), que avaliou 136.384 pessoas com idades entre 35 a 70 anos de 21 países, demonstrou que o consumo de mais de duas porções leite e derivados por dia foi associado com redução de $16 \%$ de eventos e/ou morte cardiovasculares. Segundo este mesmo estudo, indivíduos que consomem diariamente duas porções ou mais de leite e derivados apresentam risco de acidente vascular cerebral significativamente menor quando comparados com aqueles que não consumiam laticínios, entretanto, não foi encontrada redução significativa no risco de IAM com a ingestão de laticínios. No presente estudo, 64,7\% da população consumia uma porção ou menos de laticínios e 11,8\% consumia três ou mais porções diárias, sendo que 76,5\% consumia laticínios integrais.

Um estudo realizado por Kelly et al (2017) concluiu que há poucas evidências que a ingestão de cereais integrais auxilie no controle da pressão arterial diastólica e na melhora dos níveis de LDL. Já no estudo realizado por Temple (2018), ele sugere que o consumo de grãos integrais, além das fibras, possui função cardioprotetora, entretanto o consumo de carboidratos refinados, principalmente açúcar e bebidas açucaradas estão ligadas ao risco de desenvolver doença coronariana. Em nosso estudo, o consumo de alimentos pertencentes ao grupo de cereais foi inferior a seis porções diárias para 100\% dos participantes.

Quanto ao consumo de alimentos ultra processados como embutidos, salgadinhos industrializados/fritu- 
ras, 47,1\% dos indivíduos relataram consumir de duas a três vezes na semana, sendo o recomendado que o consumo destes alimentos não exceda uma vez na semana Uma pesquisa realizada no Brasil demonstrou que o consumo de alimentos ultra processados tem contribuído para a epidemia de obesidade no país. Os consumos destes alimentos influenciam num maior consumo de sódio, colesterol e gorduras. Além disso, uma pesquisa realizada no Reino Unido demonstrou que uma redução de 50\% no consumo de alimentos processados e ultra processados auxilia numa diminuição de 10 a 13\% na mortalidade cardiovascular (LOUZADA et al., 2015; MOREIRA et al., 2015).

O consumo de sódio dos indivíduos estudados foi de 2288,36mg, excedendo a recomendação diária para a população estudada. 0 consumo excessivo de sódio dietético é considerado preditor significativo de doença coronariana e eventos cardiovasculares em homens $(\mathrm{p}=0,024)$, independente da presença de hipertensão arterial sistêmica (ÄIJÄLÄ et al., 2015).

Um estudo demonstrou que o impacto da gordura saturada na saúde cardiovascular varia de acordo com a fonte alimentar, assim como os ácidos graxos monoinsaturados que apresentaram maior efeito cardioprotetor quando ingeridos na forma encontrada na dieta mediterrânea. Quanto aos ácidos graxos poli insaturados, a ingestão dietética do ômega 3 desempenha papel cardioprotetor. Em nosso estudo, os participantes apresentaram um alto consumo de gordura saturada e baixo consumo de ácidos graxos poli-insaturados (PRATES FRANKENBERG; RODRIGUES, 2015).

A alimentação dos indivíduos estudados caracterizou-se hiperprotéica, normolipídica e com um baixo teor de carboidratos de acordo do com as recomendações das Dietary Intakes. Tharrey et al. (2018)acompanharam 8337 homens e mulheres por 12 anos identificou que um aumento de 18 gramas de proteínas de origem animal aumenta em $60 \%$ a mortalidade por doenças cardiovasculares já quando esta proteínaera de origem vegetal, o risco era 40 \% menor. Além disso as maiores causas de morte foram cardiopatias isquêmicas $(37,8 \%)$, e doenças cerebrovasculares (23,7\%) (THARREY et al., 2018; FOOD AND NUTRITION BOARD et al, 2011).

A ingestão de minerais, como o cálcio, magnésio e potássio, foi abaixo do recomendado pelo Dietary Intakes. Adebamowo et al (2015), ao realizarem uma co- orte durante 24 anos com 42.669 homens entre 45 e 75 anos, puderam observar que uma dieta rica em magnésio, potássio e cálcio contribui na prevenção de acidente vascular cerebral tanto na forma isolada quanto simultânea, sendo que, quando na forma simultânea o benefício era atenuado, através do funcionamento de seus mecanismos. 0 potássio e o cálcio dietético estão associados a redução de traços isquêmicos (FOOD AND NUTRITION BOARD et al., 2011).

A vitamina B12 bem como o ácido fólico são vitaminas envolvidas no metabolismo da homocisteína, o que faz com que um baixo consumo de B12 prejudique o metabolismo da homocisteína, resultando em um quadro hiperhomocisteinemia, que está significativamente correlacionada com dislipidemias $(\mathrm{P}<0,0001)$. No presente estudo, ingestão da média da população mostrou-se deficitária, demonstrando uma maior exposição dos indivíduos ao risco de hiperhomocisteinemia (MAHALLE, 2013).

$\mathrm{O}$ déficit de Vitamina $\mathrm{D}$ bem como a hipervitaminose podem contribuir para a calcificação vascular através da alteração na sua atividade metabólica e sinalização, sendo que na Hipervitaminose, há o estímulo e diferenciação osteogênica da mineralização, o que contribui para a ocorrência de eventos cardiovasculares. 0 consumo de vitamina D, por nossa população, mostrou-se deficitário, apresentando média de 1,65mg (WANG et al., 2018).

Uma revisão de metánalises demonstrou que o consumo de fibras alimentares adequado reduz a incidência de eventos cardiovasculares, o que pode ser justificado pela redução dos níveis plasmáticos da lipoproteína da baixa densidade. A mediana de consumo de fibras no presente estudo foi de 13,45g $(10,10-24,11)$ caracterizando uma alimentação pobre em fibras (MCRAE, 2017).

A prática de exercício físico (pelo menos 150 minutos semanais) era presente por apenas $11,8 \%$ dos participantes. Uma coorte prospectiva realizada em 17 países, incluindo o Brasil, demonstrou que a prática de exercício físico regular, seja ele na forma recreativa ou não, está associado significativamente $(p=0,01)$ a redução do risco cardiovascular em indivíduos de países de baixa-média renda e de baixa renda (LEAR et al., 2017).

A bioimpedância elétrica é um excelente método de avaliação, podendo ser utilizado em diferentes condições clínicas, como obesidade, sarcopenia e câncer, consistenum método não invasivo, de fácil reprodutibilida- 
de e portátil, entretanto, uma das limitações da avaliação com a bioimpedância, é o estado de hidratação, ser um método indireto e que exige de equações específicas para cada população. (CENICCOLA, 2019)

\section{CONCLUSÃO}

Nossos resultados demonstram que os jogadores de futebol de várzea estudados apresentam um conjunto de fatores de risco que os tornam potencialmente expostos a ocorrência de eventos cardiovasculares, uma vez que apresentaram padrões alimentares deficitários em relação à micronutrientes e com uma distribuição inadequada de macronutrientes. Tal composição alimentar acaba favorecendo a ocorrência de demais fatores de risco como o aumento da circunferência de cintura, e \%GC, levando a alterações na homeostase corporal e resistência à insulina. 


\section{REFERÊNCIAS}

ADEBAMOWO, Sally N. et al. Intakes of magnesium, potassium, and calcium and the risk of stroke among men. International Journal of Stroke, [S.I.], v. 10, n. 7, p. 1093-1100, 2015.

ÄIJÄLÄ, M. et al. Dietary sodium intake and prediction of cardiovascular events. European journal of clinical nutrition, [S.I.], v. 69, n. 9, p. 1042, 2015.

BLÜHER, Matthias. Obesity: global epidemiology and pathogenesis. Nature ReviewsEndocrinology, [S.I.], v.15, p. 288298, 2019 .

CENICCOLA, Guilherme Duprat et al. Current technologies in body composition assessment: advantages and disadvantages. Nutrition, v. 62, p. 25-31, 2019.

CASTANHO, Gabriela Kaiser Fullin et al. Consumo de frutas, verduras e legumes associado à Síndrome Metabólica e seus componentes em amostra populacional adulta. Ciência \& Saúde Coletiva, [S.I.] v. 18, p. 385-392, 2013.

DEHGHAN, Mahshid et al. Association of dairy intake with cardiovascular disease and mortality in 21 countries from five continents (PURE): a prospective cohort study. The Lancet [S.I.], v. 392, n. 10161, p. 2288-2297, 2018.

DOS SANTOS, Maria Cristina Batista et al. Hábitos e perfil socioeconômico dos pacientes com doença aterosclerótica no Brasil. Revista Com. Ciências em Saúde. v.22, n. 3, p 247-256, 2011.

DYRSTAD, Sindre M. et al. Waist circumference thresholds and cardiorespiratory fitness. Journal of sport and health science, [S.I.], v.8, n.1, p.17-22, 2019.

ELLIS, Kenneth J. et al. Bioelectrical impedance methods in clinical research: a follow-up to the NIH Technology Assessment Conference. Nutrition, v. 15, n. 11-12, p. 874-880, 1999.

FOOD AND NUTRITION BOARD et al. Dietary Reference Intakes (DRIs): estimated average requirements. National Academies, [S.I.], v.1997, 2011

INSTITUTO BRASILEIRO DE GEOGRAFIA E ESTATÍSTICA. Pesquisa nacional por amostra de domicílios - PNAD. 2015.

KELLY, Sarah AM et al. Whole grain cereals for the primary or secondary prevention of cardiovascular disease. Cochrane Database of Systematic Reviews, [S.I.], n. 8, 2017.

KYLE, Ursula G. et al. Bioelectrical impedance analysis-part I: review of principles and methods. Clinical nutrition, v. 23, n. 5, p. $1226-1243,2004$

LEAR, Scott A. et al. The effect of physical activity on mortality and cardiovascular disease in 130000 people from 17 high-in- come, middle-income, and low-income countries: the PURE study. The Lancet, [S.I.], v. 390, n. 10113, p. 2643-2654, 2017.

LOUZADA, Maria Laura da Costa et al. Consumption of ultra-processed foods and obesity in Brazilian adolescents and adults. Preventive medicine, [S.I.], v. 81, p. 9-15, 2015.

LUO, Wen-Shu et al. Interaction of tobacco smoking and alcohol consumption with obesity on cardiovascular disease in a Chinese cohort. Coronary Artery Disease, 2019, v.21, n.4, pp. 372-377.

MAHALLE, Namita et al. Vitamin B12 deficiency and hyperhomocysteinemia as correlates of cardiovascular risk factors in Indian subjects with coronary artery disease. Journal of cardiology, [S.I.], v. 61, n. 4, p. 289-294, 2013.

MCRAE, Marc P. Dietary fiber is beneficial for the prevention of cardiovascular disease: an umbrella review of meta-analyses. Journal of chiropractic medicine, [S.I.], v. 16, n. 4, p. 289299, 2017.

MENDONÇA, SKD; FARIAS, J. M.; GUERREIRO, KHS. Avaliação da composição corporal e perfil alimentar em futebolistas amadores.Revista ENAF Science, [S.I.],v.11, p. 206-213, 2016.

MILLER, Victoria et al. Fruit, vegetable, and legume intake, and cardiovascular disease and deaths in 18 countries (PURE): a prospective cohort study. The Lancet, [S.I.], v. 390, n. 10107, p. 2037-2049, 2017.

MINISTÉRIO DA SAÚDE. Vigitel Brasil 2018: Vigilância de fatores de risco e proteção para doenças crônicas por inquérito telefônico: estimativas sobre frequência e distribuição sociodemográfica de fatores de risco e proteção para doenças crônicas nas capitais dos 26 estados brasileiros e no Distrito Federal em 2018. Brasília: Ministério da Saúde, 2019.

MINISTÉRIO DA SAÚDE et al. Guia alimentar para a população brasileira. Brasília: Ministério da Saúde, 2014.

MOREIRA, Patricia VL et al. Comparing different policy scenarios to reduce the consumption of ultra-processed foods in UK: impact on cardiovascular disease mortality using a modelling approach. PLoS One, [S.I.], v. 10, n. 2, 2015.

MONEGO, Estelamaris et al. Alimentos brasileiros e suas porções: um guia para avaliação do consumo alimentar. Editora Rubio, 2013.

MOTAMED, Nima etal. Conicity index and waist-to-hip ratio are superior obesity indices in predicting 10-year cardiovascular risk among men and women. Clinical cardiology, v. 38, n. 9, p. 527-534, 2015.

PACHECO, Daniel A. Quintana et al. Red meat consumption and risk of cardiovascular diseases-is increased iron load a possible link? The American journal of clinical nutrition, [S.I.], v. 107, n. 1, p. 113-119, 2018. 
PITANGA, Francisco José Gondim. Antropometria na avaliação da obesidade abdominal e risco coronariano. Revista Brasileira de Cineantropometria e Desempenho Humano, v.13, n. 3, p. 238-241, 2011

PITANGA, Francisco José Gondim; LESSA, Inês. Associação entre indicadores antropométricos de obesidade e risco coronariano em adultos na cidade de Salvador, Bahia, Brasil. Revista Brasileira de Epidemiologia, São Paulo, v.10, n.2, 2007.

PONTES, Luciano Meireles de; SOUSA, Maria do Socorro Cirilo de; LIMA, Roberto Teixeira de. Perfil dietético, estado nutricional e prevalência de obesidade centralizada em praticantes de futebol recreativo. Revista Brasileira de Medicina do Esporte, [S.I.], v. 12, n. 4, p. 201-205, 2006.

PRATES, Raquel Eccel; FRANKENBERG, Anize Delfino von; RODRIGUES, Ticiana da Costa. Dietary fatty acids and cardiovascular disease: a review. Clinical and biomedical research, Porto Alegre, v.35, n.3, p. 126-133, 2015.

QUETELET, Adolphe. Anthropométrie ou mesure des différentes facultés de l'homme. Brussels: C. Muquardt, 1870.

SIPPEL, Crislene Aschebrock et al. Processos inflamatórios da obesidade. Revista de Atenção à Saúde, [S.I.], v. 12, n. 42, 2014.

SOCIEDADE BRASILEIRA DE CARDIOLOGIA (SBC). Cardiôme-
SOCIEDADE DE CARDIOLOGIA DO RIO DE JANEIRO (SCERJ). Doença Coronariana. Rio de Janeiro: SOCERJ, 2013. Disponível em: <https://socerj.org.br/doenca-coronariana/>. Acesso em: 18 novembro 2018.

TEMPLE, Norman J. Fat, sugar, whole grains and heart disease: 50 years of confusion. Nutrients, [S.I.], v. 10, n. 1, p. 39, 2018.

TOMÁS, Rita; BRITO, João. 0 futebol recreativo na promoção da Atividade Física em todas as idades. Revista Factores de Risco, Lisboa, v.44, p. 98-105, abr. 2017.

THARREY, Marion et al. Patterns of plant and animal protein intake are strongly associated with cardiovascular mortality: the Adventist Health Study- 2 cohort. International journal of epidemiology, Raleigh, v.47, n.5, p.1603-1612, 2018.

WANG, Jeffrey et al. Vitamin D in vascular calcification: a double-edged sword?. Nutrients, [S.I.], v. 10, n. 5, p. 652, 2018. WORLD HEALTH ORGANIZATION (WHO). Enfermedades cardiovasculares. 2015. Disponível em: <http://www.who.int/ mediacentre/factsheets/fs317/es/>. Acesso em: 03 out 2017.

WORLD HEALTH ORGANIZATION. Obesity: preventing and managing the global epidemic: report of a WHO consultation on obesity. Geneva: World Health Organization, 1998

ZHANG, Xiangyu et al. High-protein diets increase cardiovascular risk by activating macrophage mTOR to suppress mitophagy. Nature Metabolism, [S.I.], v. 2, n. 1, p. 110-125, 2020. 